
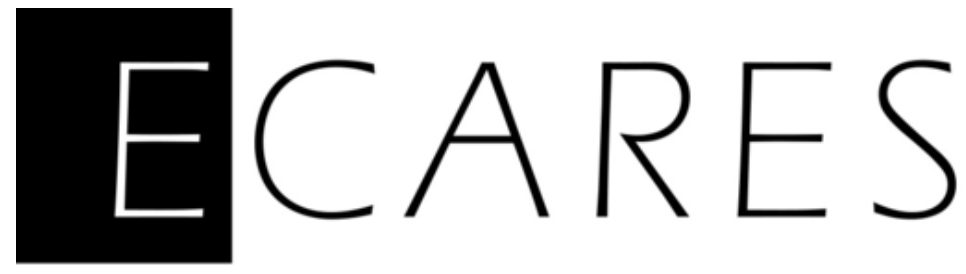

\title{
Measuring Competitiveness and Cooperativeness
}

\author{
Thomas Demuynck \\ SBS-EM, ECARES, Université libre de Bruxelles \\ Christian Seel \\ Department of Economics, Maastricht University \\ Giang Tran \\ Department of Economics, Maastricht University, Maastricht
}

May 2019 


\title{
Measuring competitiveness and cooperativeness
}

\author{
Thomas Demuynck * Christian Seel ${ }^{\dagger} \quad$ Giang Tran ${ }^{\ddagger}$
}

May 2, 2019

\begin{abstract}
We develop an index of competitiveness and cooperativeness which is based on the primitives of a normal-form game, i.e., players, strategies and payoffs. The index relies on a unique decomposition of a given game into a zero-sum game and a common-interest game. The index decreases in the distance to its zero-sum part and it increases in the distance to its common-interest part. Alternatively, the index increases if the share of variation in payoffs captured by the zero-sum part increases. We compute our index for well-known classes of games such as Prisoner's Dilemma, games with Strategic Complements and Substitutes, All-pay auctions, Tullock contests, and Public Goods games. The comparative statics of our index coincide with economic intuition. The index does well in explaining experimental findings in the sense that more cooperative and less competitive behavior correlates with lower values of the index.

KEYWORDS: Competitiveness, cooperativeness, index

JEL-ClassificATION: Cr2.
\end{abstract}

${ }^{*}$ Ecares, Université Libre de Bruxelles, Belgium. E-Mail: Thomas.Demuynck@ulb.ac.be. Thomas Demuynck acknowledges financial support by the Fonds de la Recherche Scientifique-FNRS under grant nr F.4516.18

${ }^{\dagger}$ Department of Economics, Maastricht University, Maastricht, The Netherlands. E-Mail: C.Seel@maastrichtuniversity.nl

‡Department of Economics, Maastricht University, Maastricht, The Netherlands. E-Mail: Giang.Tran@maastrichtuniversity.nl 


\section{Introduction}

The emergence of cooperation in strategic situations has been studied in different literatures, both within and beyond economics. For instance, a central question in the literature on repeated games asks under which conditions cooperative behavior be sustained as a (subgame-perfect) equilibrium. In biology and evolutionary game theory, a central topic of interest is the emergence of altruistic behavior, where an individual sacrifices part of her own payoff to improve overall welfare. Similarly, many studies in behavioral psychology analyze drivers of prosocial behavior. At the same time, other literatures study different notions of competition. In industrial organization, competition is often measured by comparing the market or equilibrium outcome to the outcome under perfect competition. This leads to indices based on firms' market shares, like the Herfindahl-Hirschman index or firms' mark-ups, like the Lerner index. The literature on gender differences initiated by Gneezy, Niederle and Rustichini (2003) finds that women often respond less favorably to competition than men and are less likely to select into competition. Here, the differentiation in terms of competition is based on whether relative or absolute performance is rewarded. While these various notions of cooperation and competition are very useful, they are also particular to the setting under consideration.

Despite the wide interest for notions of cooperation and competition in the literature, there exists, to the best of our knowledge, no universal index measuring the competitiveness and cooperativeness for a given environment. The main goal of this paper is to develop such an index. Towards this end, we start from the setting of a normal-form game. Our index is easy to compute and only uses the primitives of the game, i.e., players, strategies and payoffs. Moreover, the index accommodates both finite and infinite strategy spaces.

In order to study the notions of cooperativeness and competitiveness we start from two benchmarks environments. Using the language of game theory, we believe the most 'competitive' setting is captured by a zero-sum game whereas the most 'cooperative' setting corresponds to a common-interest game (i.e. when both players have identical payoffs). Zero-sum games, often called strictly competitive games, have the feature that the agents' payoffs sum to zero, i.e., the gain or loss for one agent is exactly balanced by the loss or gain of the other agents. On the other hand, for common interest games, the payoffs of all agents are identical. In this case, there is no conflict of interest among the various players. This categorization motivates a main ingredient of our index, which is the decomposition of a game into a 'competitive' zero-sum part and a 'cooperative' common-interest part. For two players and finitely many strategies, this decomposition was introduced in Kalai and Kalai (2013). ${ }^{1}$ With the help of tools from linear algebra, we extend the decomposition to an arbitrary number of players and to infinite strategy spaces. We also show that the decomposition is unique. Our index is constructed such that it increases, when a game is closer to its zero-sum part and farther away from its common-interest part. As such, higher values of our index indicate more competitiveness (or less cooperativeness). We

\footnotetext{
${ }^{1}$ Kalai and Kalai (2013) use the decomposition to define a semi-cooperative solution, the coco value, for games with transferable utility.
} 
provide two interpretations for our index, a geometric and a statistical interpretation.

For the geometric interpretation, the index is constructed in two steps. In step one, we subtract from all players' payoffs their mean payoff (over all strategy profiles). Denoting by $g$ the game and by $\bar{g}$ the game where the payoff of each player is constant at its average payoff in $g$, this defines a new normal-form game $g^{*}=g-\bar{g}$ where the average payoff of every player equals zero. ${ }^{2}$ The normalized game $g^{*}$ is invariant under the addition of a constant to the payoffs of a particular player in $g$. This property is ubiquitous in game theory. Depending on the exact topic, it has different names such as best-reply invariance (Harsanyi and Selten, 1988), invariance under local shifts in evolutionary game theory (Weibull, 1995) or adding a dummy game in the literature on potential games (Facchini, van Megen, Borm, and Tijs, 1997).

We can decompose the normalized game $g^{*}$ into its zero-sum part $z^{*}$ and its commoninterest part $c^{*}$, i.e., $g^{*}=z^{*}+c^{*}$. Our competitiveness-cooperativeness index CCI takes the form:

$$
\mathrm{CCI}=\frac{\left\|g^{*}-c^{*}\right\|^{2}}{\left\|g^{*}\right\|^{2}}=1-\frac{\left\|g^{*}-z^{*}\right\|^{2}}{\left\|g^{*}\right\|^{2}},
$$

where $\|$.$\| is a suitably defined norm obtained from an inner product on the space of all$ games. The identity above is obtained from the fact that $\left\|g^{*}\right\|^{2}=\left\|z^{*}\right\|^{2}+\left\|c^{*}\right\|^{2}$ (see Section 3). If payoffs are perfectly aligned, i.e., the underlying game is a common-interest game, one can show that this ratio equals zero as in this case $g^{*}=c^{*}$. If $g$ is a zero-sum game then $g^{*}=z^{*}$ so the index equals 1 . More generally, our index takes values between 0 and 1 where values are closer to one if the normalized game $g^{*}$ is closer to its zero-sum component $z^{*}$. We provide a simple axiomatization of our index.

Our index also has a statistical interpretation. Denoting by $z$ the zero-sum part of a game $g$, we show that

$$
\mathrm{CCI}=\frac{\|z-\bar{z}\|^{2}}{\|g-\bar{g}\|^{2}} .
$$

Here, the index can be interpreted as measuring the fraction of the variance in payoffs in a normal-form game $\|g-\bar{g}\|^{2}$ that is captured by the variance in payoffs of its zero-sum component $\|z-\bar{z}\|^{2}$. In this sense, the index has a similar interpretation as an $R^{2}$-statistic in a linear regression: it tells us how much of the variation in the payoffs of $g$ is explained by its non-cooperative zero-sum part $z$.

We see at least three settings in which our index could be useful. First, by providing a single index that can be universally applied to normal-form games, our index can replace ad-hoc reasoning about which payoff changes make it more likely to observe a cooperative or competitive outcome in a particular game. For instance, such reasoning is encountered in the literature on the Prisoner's Dilemma with so-called 'temptation payoffs', which relates to the benefit of deviating from the Pareto efficient profile and 'punishment payoffs', which refers to the payoff in the dominant-strategy equilibrium. Given that our index produces a single number, it enables us to disentangle settings where more cooperation is to be

\footnotetext{
${ }^{2}$ A similar idea of filtering out non-strategic parts appears in Candogan, Menache, Ozdaglar and Parrilo (2011) who decompose games into a non-strategic, a potential and a harmonic component.
} 
expected even when multiple payoffs are changed simultaneously. While we calculate the index using a normal-form game, it can also be used for comparative static predictions for payoff changes in the stage game of a repeated game. Since our approach is entirely non-procedural, we see it as complementary to other approaches which study effects on cooperation based on the temporal structure (e.g., Roth and Murnighan, 1978 for the repeated Prisoner's Dilemma).

Second, our index can be useful as input for the recent experimental literature claiming that after facing a more competitive environment, subjects tend to behave less cooperatively in the future (Buser and Dreber, 2016; Carpenter and Seki, 2006; Peysakhovich and Rand, 2015). In such settings, our index allows the experimenter to formally classify games according to their competitiveness. Thus, she can test how future behavior depends on the value of our index of competitiveness.

Third, our index could be useful in the literature on gender differences. In particular, one might test for a large class of different games if women tend to select into less competitive games compared to men. To do so, one could calculate the index of different parametrizations of a given game and let participants decide which game they prefer to play. Our conjecture is that, on average, women tend to select into the less competitive parametrizations.

In this paper, we mainly follow the first setting for using our index, i.e., we see it as a comparative static predictor of competitive versus cooperative behavior. For this purpose, the second part of the paper derives and computes our index for a sample of games that includes the Prisoner's Dilemma, the Battle of the Sexes, games with Strategic Complements and Substitutes, All-pay auctions, Tullock contests, and Public Good games. This sample is chosen to (i) consist of games which have been extensively studied in the literature, either with a focus on cooperativeness or competitiveness and (ii) to cover settings with both finite and infinite strategy spaces.

For each game, we contrast the values obtained from our index with the experimental findings from the literature. We find that our index provides a good general indicator when a game is more or less competitive, both in line with economic intuition and experimental findings, with some idiosyncratic nuances for variations in the experimental setup. Although the selection of studies that we discuss is far from exhaustive, we tried our best to give a proper account of the experimental literature and use meta-studies if available. Let us remark, however, that our analysis requires to only include studies where our measure changes between the different treatments; and without any variation in the number of players or the set of actions.

The rest of the paper is organized as follows. Section 2 presents a motivating example. Section 3 introduces our index and provides an axiomatization of our index for games with finite strategy spaces. We apply and discuss the index for games with finite strategy spaces in Section 4 and for games with infinite strategy spaces in Section 5. Section 6 provides a brief discussion. In Appendix A, we extend the theoretical framework to games with infinite strategy spaces and we collect the proofs of the theory section. We provide more details on the applications in Appendix B. 


\section{Motivating example}

As a motivating example, let $b>1$ and consider the following two-player Prisoner's Dilemma game:

\begin{tabular}{cc|cc} 
& & Player 2 & \\
& & $\mathrm{C}$ & $\mathrm{D}$ \\
\hline Player 1 & $\mathrm{C}$ & $(1,1)$ & $(-b, b)$ \\
& $\mathrm{D}$ & $(b,-b)$ & $(-1,-1)$
\end{tabular}

Both players have two strategies: $\mathrm{D}$ (efect) or $\mathrm{C}$ (ooperate). Note $(\mathrm{C}, \mathrm{C}$ ) is the maximizes the joint payoffs and Pareto dominates (D,D), which is the Nash unique equilibrium in dominant strategies. ${ }^{3}$

In the experimental literature on Prisoner's Dilemma games, there are two key features that make (C,C) less likely to be chosen. First, there is the 'temptation' incentive which can be measured as the difference between the cooperative outcome 1 and the payoff $b$ that is obtained by a unilateral deviation. Next there is the 'punishment' incentive, which is related to the difference between the so called 'sucker payoff' $-b$ obtained when you are the only player that is cooperating and the payoff -1 that is obtained when both players defect. In the above example, both incentives increase with the value of $b$. So higher values of $b$ should make the outcome $(\mathrm{C}, \mathrm{C})$ less likely to be observed.

The above game can be summarized as $g=(A, B)$ where $A$ is the payoff matrix of player $1, A=\left[\begin{array}{ll}1 & -b \\ b & -1\end{array}\right]$, and $B$ is the payoff matrix of player $2, B=\left[\begin{array}{cc}1 & b \\ -b & -1\end{array}\right]$. Elementary calculations show that the payoff matrices $A$ and $B$ can be decomposed in the following way:

$$
\begin{aligned}
& A=\frac{A-B}{2}+\frac{A+B}{2}, \\
& B=\frac{B-A}{2}+\frac{A+B}{2} .
\end{aligned}
$$

For the game above, we obtain:

$$
\underbrace{\left[\begin{array}{cc}
(1,1) & (-b, b) \\
(b,-b) & (-1,-1)
\end{array}\right]}_{g}=\underbrace{\left[\begin{array}{cc}
(0,0) & (-b, b) \\
(b,-b) & (0,0)
\end{array}\right]}_{z_{g}}+\underbrace{\left[\begin{array}{cc}
(1,1) & (0,0) \\
(0,0) & (-1,-1)
\end{array}\right]}_{c_{g}}
$$

The first game on the right hand side is a zero-sum game $z_{g}=((A-B) / 2,(B-A) / 2)$ while the second game is a common-interest game $c_{g}=((A+B) / 2,(A+B) / 2)$. As we will show below, this decomposition into a zero-sum part and common-interest part is unique. Zero-sum games are commonly seen as being the most competitive class of games

\footnotetext{
${ }^{3}$ In the example, the average payoff of each player equals zero. As will be explained in the next section, if average payoffs are not zero, we should first perform a mean normalization.
} 
while common-interest games are the most cooperative. Intuitively, interests are perfectly opposite in the former class while interests are perfectly aligned in the latter class.

The idea behind the construction of our index is to measure the competitiveness of a game $g$ using its distance to its zero-sum component $z_{g}$ versus its distance to the commoninterest part $c_{g}$. The farther away $g$ is from $z_{g}$ and the closer $g$ is to $c_{g}$, the less competitive and the more cooperative $g$ is.

In order to measure distance between games, we use norms. In particular, for a matrix $A$, let $\|A\|$ be the Frobenius norm of $A$, i.e., $\|A\|^{2}=\sum_{i, j}\left(a_{i, j}\right)^{2}$ where $a_{i, j}$ is the value on row $i$ and column $j$ of matrix $A$. For a game $g=(A, B)$ let us then define the norm $\|g\|^{2}=\|A\|^{2}+\|B\|^{2}$. Interestingly (see Lemma 1 below), it is possible to show that, for the decomposition above:

$$
\|g\|^{2}=\left\|z_{g}\right\|^{2}+\left\|c_{g}\right\|^{2}
$$

If $\|g\|^{2} \neq 0$, then it follows from $g=z_{g}+c_{g}$ that:

$$
1=\frac{\left\|g-c_{g}\right\|^{2}}{\|g\|^{2}}+\frac{\left\|g-z_{g}\right\|^{2}}{\|g\|^{2}} .
$$

The 'closer' $g$ is to the zero-sum game $z_{g}$, the larger is the first term and the smaller is the second term, while both terms always sum to 1 . We use the first term to define our index of competitiveness:

$$
\mathrm{CCI}=\frac{\left\|g-c_{g}\right\|^{2}}{\|g\|^{2}} \equiv \frac{\left\|g-c_{g}\right\|^{2}}{\left\|g-c_{g}\right\|^{2}+\left\|g-z_{g}\right\|^{2}} .
$$

This index always takes values between zero and one. It equals one if $g$ is a zero-sum game and it equals zero if $g$ is a common-interest game. For the particular Prisoner's Dilemma game above, our index equals:

$$
\mathrm{CCI}=\frac{(-b)^{2}+b^{2}+b^{2}+(-b)^{2}}{(-b)^{2}+b^{2}+b^{2}+(-b)^{2}+1^{2}+1^{2}+1^{2}+1^{2}}=\frac{b^{2}}{1+b^{2}},
$$

which is increasing $b$. Intuitively, we would expect that a higher index of competitiveness, i.e., higher values of $b$, makes it less likely to observe a cooperative outcome.

\section{Main results}

In this section we outline the main derivation of our index of competitiveness or cooperativeness. The more technical details are relayed to Appendix A.

\subsection{Setting}

We consider a setting with a finite number of players $\{1, \ldots, N\}$. Each player $i \leq N$ has a set of strategies (actions) $S_{i}$ which might be finite or infinite. We denote the product of 
the strategy sets by $S=\prod_{i=1}^{N} S_{i}$. Each player $i \leq N$ has a payoff function $\pi_{i}: S \rightarrow \mathbb{R}$ that associates a real number to every strategy profile. Here, we interpret payoffs in a cardinal sense, i.e., if two games have different payoff functions, then they are different.

A game $g$ then consists of a set of players $N$, a set of strategies $S_{i}$ for each player $i$, and payoff functions $\pi_{i}: S \rightarrow \mathbb{R}$. We denote by $\mathcal{G}$ the set of all games with $N$ players and strategy sets $\left(S_{i}\right)_{i \leq N}$. From now on, we fix the set of players and the strategy sets. We therefore omit the dependence on $\left(S_{i}: i \leq N\right)$ in the notation. Thus, we denote a game $g$ with payoff functions $\pi_{i}: S \rightarrow \mathbb{R}$ by $g=\left\{\pi_{i}: i \leq N\right\}$.

For two games $g=\left\{\pi_{i}: i \leq N\right\}$ and $\tilde{g}=\left\{\tilde{\pi}_{i}: i \leq N\right\}$ in $\mathcal{G}$, we can define the game $g+\tilde{g} \in \mathcal{G}:$

$$
g+\tilde{g}=\left\{\pi_{i}+\tilde{\pi}_{i}: i \leq N\right\} .
$$

Next, for all games $g=\left\{\pi_{i}: i \leq N\right\} \in \mathcal{G}$ and $\alpha \in \mathbb{R}$, we can define the game $\alpha g \in \mathcal{G}$ :

$$
\alpha g=\left\{\alpha \pi_{i}: i \leq N\right\}
$$

Since $\mathcal{G}$ is closed under addition and scalar multiplication, we have that $\mathcal{G}$ is a vector space. The zero element is $g_{0}=\left\{\pi_{i}^{0}: i \leq N\right\}$ where for all $i \leq N$ and $s \in S, \pi_{i}^{0}(s)=0$.

By defining a suitable inner product, ${ }^{4}$ we can turn $\mathcal{G}$ into a Hilbert space, i.e., a normed vector space. For example, if the set of strategy profiles $S$ is finite, we might define the inner product between the games $g=\left\{\pi_{i}: i \leq N\right\}$ and $\tilde{g}=\left\{\tilde{\pi}_{i}: i \leq N\right\}$ by:

$$
\langle g, \tilde{g}\rangle=\sum_{i=1}^{N} \sum_{s \in S} \pi_{i}(s) \tilde{\pi}_{i}(s) .
$$

As usual for Hilbert spaces, we define the squared norm $\|\cdot\|^{2}$ by $\|g\|^{2}=\langle g, g\rangle$, so $\|g\|^{2}=$ $\langle g, g\rangle=\sum_{i=1}^{N} \sum_{s \in S}\left(\pi_{i}(s)\right)^{2}$. The function $d(g, \tilde{g})=\|g-\tilde{g}\|$ is a metric on the space $\mathcal{G}$.

Technically, when defining an inner product and the resulting norm, $\|$.$\| , it is possible$ to give more weight to certain strategy profiles compared to others or (in the limit) to even rule out some strategy profiles from consideration. ${ }^{5}$ We believe, however, that a proper model or experiment should only include strategies of interest. Since our index is silent about the temporal structure, we do not think that ruling out strategies (e.g., the ones that are dominated in a one-shot game) is a desirable feature in general. In particular, such strategies might be part of a subgame-perfect equilibrium in a repeated game and, as we know from the experimental literature, they might even be observed in a one-shot game.

Under some conditions on the payoff functions, it is possible to define inner products for classes of games with infinite strategy spaces. For ease of exposition, this section will focus on finite games where the inner product is defined as in Eq. (1). We refer to Appendix A for a derivation of our results for more general inner products that also allow some strategy

\footnotetext{
${ }^{4}$ An inner product $\langle.,$.$\rangle on \mathcal{G}$ is a function from $\mathcal{G} \times \mathcal{G}$ to $\mathbb{R}$ that is bilinear, i.e., for $g, \tilde{g}, \hat{g}, g^{\prime} \in \mathcal{G}$, $\left\langle\alpha g+\beta \tilde{g}, \gamma \hat{g}+\delta g^{\prime}\right\rangle=\alpha \gamma\langle g, \hat{g}\rangle+\alpha \delta\left\langle g, g^{\prime}\right\rangle+\beta \gamma\langle\tilde{g}, \hat{g}\rangle+\beta \delta\left\langle\tilde{g}, g^{\prime}\right\rangle$.

${ }^{5}$ Technically, in this case, we should redefine the vector space to be the set of equivalence classes of games, where $g$ and $g^{\prime}$ are in the same equivalence class if $\left\|g-g^{\prime}\right\|=0$.
} 
profiles to receive higher weight than others. This includes settings with infinite strategy spaces as discussed in Section 5.

\subsection{The competitiveness-cooperativeness index}

The aim of this section is to derive an index CCI $: \mathcal{G} \rightarrow \mathbb{R}$ such that $\mathrm{CCI}(g)$ gives an index of competitiveness (or cooperativeness) for the normal-form game $g$. Then, for two games $g, g^{\prime} \in \mathcal{G}, \mathrm{CCI}(g)>\mathrm{CCI}\left(g^{\prime}\right)$ is an indication that game $g$ is more competitive and less cooperative than game $g^{\prime}$.

Decompositions In this paragraph we will start by defining several subclasses of games and show how a game can be decomposed into members of these classes. We denote by $\mathcal{K}$ the class of games in $\mathcal{G}$ for which each player has constant payoffs:

$$
\mathcal{K}=\left\{g=\left\{\pi_{i}: i \leq N\right\} \in \mathcal{G}: \forall s, s^{\prime} \in S, \pi_{i}(s)=\pi_{i}\left(s^{\prime}\right)\right\}
$$

The second class of games $\mathcal{M}$ consists of the games for which the average payoff of each player equals zero:

$$
\mathcal{M}=\left\{g=\left\{\pi_{i}: i \leq N\right\} \in \mathcal{G}: \frac{1}{|S|} \sum_{s \in S} \pi_{i}(s)=0\right\},
$$

where $|S|$ is the cardinality of the set $S$. The two sets $\mathcal{K}$ and $\mathcal{M}$ are orthogonal in the sense that for $g \in \mathcal{K}$ and $\tilde{g} \in \mathcal{M},\langle g, \tilde{g}\rangle=0$. Indeed, if $g=\left\{\pi_{i}: i \leq N\right\} \in \mathcal{K}$ and $\tilde{g}=\{\tilde{\pi}: i \leq N\} \in \mathcal{M}$, then for any $s_{0} \in S$ :

$$
\begin{aligned}
\langle g, \tilde{g}\rangle & =\sum_{i=1}^{N} \sum_{s \in S} \pi_{i}(s) \tilde{\pi}_{i}(s), \\
& =\sum_{i=1}^{N} \sum_{s \in S} \pi_{i}\left(s_{0}\right) \tilde{\pi}_{i}(s), \\
& =\sum_{i=1}^{N} \pi_{i}\left(s_{0}\right) \sum_{s \in S} \tilde{\pi}_{i}(s)=0 .
\end{aligned}
$$

The second equality follows from the fact that for all $s \in S$ and $i \leq N, \pi_{i}(s)=\pi_{i}\left(s_{0}\right)$. The last equality follows from the fact that $\tilde{g} \in \mathcal{M}$. It is easy to verify that both $\mathcal{K}$ and $\mathcal{M}$ are vector spaces (i.e., that they are closed under addition and multiplication). Consider the following projections: ${ }^{6}$

$$
\begin{aligned}
& K g=\arg \min _{\tilde{g} \in \mathcal{K}}\|g-\tilde{g}\|^{2}, \\
& M g=\arg \min _{\tilde{g} \in \mathcal{M}}\|g-\tilde{g}\|^{2} .
\end{aligned}
$$

\footnotetext{
${ }^{6}$ As usual in linear algebra, we drop the brackets in the notation, e.g., we denote $K(g)$ by $K g$.
} 
As both $\mathcal{M}$ and $\mathcal{K}$ are vector spaces, the projections $K$ and $M$ are linear, i.e., $M(g+\beta \tilde{g})=$ $M g+\beta M \tilde{g}$ and $K(g+\beta \tilde{g})=K g+\beta K \tilde{g}$. As we will show below, every game $g$ can be uniquely decomposed as a sum of a game in $\mathcal{K}$ and a game in $\mathcal{M}$, in particular,

$$
g=K g+M g .
$$

In addition to the classes $\mathcal{M}$ and $\mathcal{K}$ we consider two other classes of games. The first, $\mathcal{Z}$, is the class of zero-sum games:

$$
\mathcal{Z}=\left\{g=\left\{\pi_{i}: i \leq N\right\} \in \mathcal{G}: \forall s \in S, \sum_{i=1}^{N} \pi_{i}(s)=0\right\} .
$$

The second, $\mathcal{C}$, is the class of common-interest games:

$$
\mathcal{C}=\left\{g=\left\{\pi_{i}: i \leq N\right\} \in \mathcal{G}: \forall s \in S, \forall i, j \leq N, \pi_{i}(s)=\pi_{j}(s)\right\} .
$$

Both $\mathcal{Z}$ and $\mathcal{C}$ are vector spaces (i.e., closed under addition and scalar multiplication) and $\mathcal{Z}$ is orthogonal to $\mathcal{C}$, i.e., for all $g \in \mathcal{Z}$ and all $\tilde{g} \in \mathcal{C}$, we have $\langle g, \tilde{g}\rangle=0$. Similar to the projections $K$ and $M$ above, we can define linear projections for $\mathcal{Z}$ and $\mathcal{C}$ :

$$
\begin{aligned}
& Z g=\min _{\tilde{g} \in \mathcal{Z}}\|g-\tilde{g}\|^{2}, \\
& C g=\min _{\tilde{g} \in \mathcal{C}}\|g-\tilde{g}\|^{2} .
\end{aligned}
$$

Given a vector space $\mathcal{A} \subseteq \mathcal{G}$, the orthogonal space $\mathcal{A}^{\perp}$ is defined as the set of all games $\tilde{g}$ such that for all $g \in \mathcal{A}$, we have $\langle g, \tilde{g}\rangle=0$. The following lemma gathers several interesting facts about the classes and operators defined in this paragraph.

\section{Lemma 1.}

1. $\mathcal{M}^{\perp}=\mathcal{K}$ and $\mathcal{Z}^{\perp}=\mathcal{C}$.

2. Every game $g$ can be uniquely decomposed as

$$
g=\tilde{g}+\hat{g},
$$

where $\tilde{g} \in \mathcal{M}$ and $\hat{g} \in \mathcal{K}$. In particular $\tilde{g}=M g$ and $\hat{g}=K g$. Moreover, in this case,

$$
\|g\|^{2}=\|M g\|^{2}+\|K g\|^{2} .
$$

3. Every game g can be uniquely decomposed as

$$
g=\tilde{g}+\hat{g},
$$

where $\tilde{g} \in \mathcal{Z}$ and $\hat{g} \in \mathcal{C}$. In particular $\tilde{g}=Z g$ and $\hat{g}=C g$. Moreover, in this case,

$$
\|g\|^{2}=\|Z g\|^{2}+\|C g\|^{2} .
$$


4. For $g=\left\{\pi_{i}: i \leq N\right\}$ we have that

- $K g=\left\{\tilde{\pi}_{i}: i \leq N\right\}$ is defined by, $\tilde{\pi}_{i}(s)=\frac{1}{|S|} \sum_{s \in S} \pi_{i}(s)$.

- $C g=\left\{\tilde{\pi}_{i}: i \leq N\right\}$ is defined by, $\tilde{\pi}_{i}(s)=\frac{1}{N} \sum_{j \leq N} \pi_{j}(s)$.

5. The operators $(M, Z),(M, C),(K, Z)$ and $(K, C)$ are commutative. In other words, for all $g \in \mathcal{G}, M Z g=Z M g, K Z g=Z K g, M C g=C M g$, and $K C g=C K g$.

The first part of Lemma 1 states that the orthogonal space of $\mathcal{M}$ equals $\mathcal{K}$ and the orthogonal space of $\mathcal{Z}$ equals $\mathcal{C}$. Parts 2 and 3 are a formalization of a well-known result in linear algebra that any element can be uniquely decomposed into its orthogonal components. Part 4 gives a closed form expression for the $\mathcal{K}$ and $\mathcal{C}$ projections of a game. The first is obtained by giving to each player the average payoff over all strategy profiles. The second is obtained by giving each player the average payoff over all players. Finally, Part 5 shows that the operators $(M, K)$ and $(Z, C)$ are commutative. This means that first applying $K(M)$ and then $Z(C)$ to a game or first applying $Z(C)$ and then $K(M)$ to a game will lead to the same result.

Consider a game $g=\left\{\pi_{i}: i \leq N\right\}$. If we combine parts 2, 3 and 4 of Lemma 1, we also obtain a closed form expression for the games $Z g=\{\tilde{\pi}: i \leq N\}$ and $M g=\{\hat{\pi}: i \leq N\}$ :

$$
\begin{aligned}
& \tilde{\pi}_{i}(s)=\pi_{i}(s)-\frac{1}{N} \sum_{j \leq N} \pi_{j}(s), \\
& \hat{\pi}_{i}(s)=\pi_{i}(s)-\frac{1}{|S|} \sum_{s \in S} \pi_{i}(s) .
\end{aligned}
$$

Axiomatization and geometric interpretation In the next step, we derive our competitiveness-cooperativeness index $\mathrm{CCI}: \mathcal{G} \rightarrow \mathbb{R}$ by imposing several conditions. As a first assumption, we require the index to remain invariant if we add a common value to all payoffs of a certain player. Formally, let $n_{i}=\left\{\pi_{j}: j \leq N\right\}$ be the game where for all strategies $s \in S, \pi_{i}(s)=1$ and for all strategies $s \in S$ and agents $j \neq i, \pi_{j}(s)=0$.

Assumption 1 (Normalization). For all $g \in \mathcal{G}$, all $\alpha \in \mathbb{R}$ and all $i \leq N$,

$$
\mathrm{CCI}\left(g+\alpha n_{i}\right)=\operatorname{CCI}(g) \text {. }
$$

The idea behind Assumption 1 is that the competitiveness of a game should not vary if we change the endowments of the players, i.e., should not exhibit any income effects. From Lemma 1 one can easily derive that:

$$
K g=\sum_{i \leq N} \frac{\left\langle n_{i}, g\right\rangle}{\left\langle n_{i}, n_{i}\right\rangle} n_{i}
$$

For a given player $i$, we can set $\alpha=-\frac{\left\langle n_{i}, g\right\rangle}{\left\langle n_{i}, n_{i}\right\rangle}$ in Assumption 1. When we repeat this step for each player, then for all $g \in \mathcal{G}$, we obtain:

$$
\mathrm{CCI}(g)=\mathrm{CCI}(g-K g)=\mathrm{CCI}(M g),
$$


where we used the decomposition $g=K g+M g$ (see Lemma 1).

As a second assumption, we require that our index should be invariant to scalar multiplication of the underlying game. In other words, if we multiply all payoffs of all players by a common factor, then the index of should not change.

Assumption 2. For all $g \in \mathcal{G}$ and all $\alpha>0$,

$$
\mathrm{CCI}(\alpha g)=\mathrm{CCI}(g)
$$

Note that the transformation in Assumption 2 multiplies the payoffs of all players by the same factor. This is distinct from a transformation where the factors may vary over the players, which would transform a game $g=\left\{\pi_{i}: i \leq N\right\}$ into a game $\tilde{g}=\left\{\alpha_{i} \pi_{i}: i \leq N\right\}$ $\left(\alpha_{i}>0\right)$ where the values $\alpha_{i}$ may differ across agents. In general, our final measure will not be invariant under such stronger transformations. Our viewpoint is that although one can make a strong argument that the competitiveness should not change under common rescaling of all payoffs, e.g., changing the denomination of the payoffs from $\$$ to $€$, this is less so when the scaling factors are agent specific. Consider, for example, the following two games:

\begin{tabular}{l|ll} 
& $\mathrm{C}$ & $\mathrm{D}$ \\
\hline $\mathrm{C}$ & 2,2 & $-1,3$ \\
$\mathrm{D}$ & $3,-1$ & 0,0
\end{tabular}

\begin{tabular}{l|ll} 
& $\mathrm{C}$ & $\mathrm{D}$ \\
\hline $\mathrm{C}$ & 20,2 & $-10,3$ \\
$\mathrm{D}$ & $30,-1$ & 0,0
\end{tabular}

The left game is a standard Prisoners' Dilemma game where the cooperative outcome (C,C) both Pareto dominates the equilibrium profile (D,D) and maximizes the joint payoffs. Both features advocate $(\mathrm{C}, \mathrm{C}$ ) as the 'cooperative' outcome. On the other hand, consider the game on the right side, which is obtained by multiplying all payoffs of player 1 by 10 . Although $(\mathrm{C}, \mathrm{C})$ still Pareto dominates $(\mathrm{D}, \mathrm{D})$, it no longer maximizes the joint payoff: the joint payoff of $(\mathrm{C}, \mathrm{C})$ is now 22 while the joint payoff of the profile $(\mathrm{D}, \mathrm{C})$ is 29 . In this perspective, for the right game, we could also consider the profile $(\mathrm{D}, \mathrm{C})$ to be a potential 'cooperative' outcome (as it maximizes the joint payoffs). If one feels that such change of the joint-payoff maximal profile might influence the competitiveness of the game, then the measure should not be invariant to an agent specific rescaling of the payoffs. Notice that this issue does not arise when we limit the transformations to the ones in Assumptions 1 or 2 .

As a final key assumption, we require that for any two games in $\mathcal{M}$, if the first game is closer to its zero-sum component and farther away from its common-interest component compared to the second game, then its index of competitiveness must be greater.

Assumption 3. Let $g, \tilde{g} \in \mathcal{M}$. If

$$
\|g-Z g\| \leq\|\tilde{g}-Z \tilde{g}\| \text { and }\|g-C g\| \geq\|\tilde{g}-C \tilde{g}\|
$$

then $\mathrm{CCI}(g) \geq \mathrm{CCI}(\tilde{g})$ with a strict inequality if in addition either $\|g-Z g\|<\|\tilde{g}-Z \tilde{g}\|$ or $\|g-C g\|>\|\tilde{g}-C \tilde{g}\|$. 
Assumptions 1-3 allow us to establish the following characterization.

Proposition 1. Assumptions 1-3 are necessary and sufficient conditions such that for all $g, \tilde{g} \in \mathcal{G} \backslash \mathcal{K}, \mathrm{CCI}(g) \geq \mathrm{CCI}(\tilde{g})$ if and only if:

$$
\frac{\|Z M g\|^{2}}{\|M g\|^{2}} \geq \frac{\|Z M \tilde{g}\|^{2}}{\|M \tilde{g}\|^{2}}
$$

This proposition shows that for $g \in \mathcal{G} \backslash \mathcal{K}$ the index CCI is a monotone transformation of $\|Z M g\|^{2} /\|M g\|^{2}$. Given this, we simply define, for all $g \in \mathcal{G} \backslash \mathcal{K}$ :

$$
\mathrm{CCI}(g)=\frac{\|Z M g\|^{2}}{\|M g\|^{2}}=\frac{\|M g-C M g\|^{2}}{\|M g\|^{2}} .
$$

This still leaves unspecified the values of $\mathrm{CCI}(g)$ for games $g \in \mathcal{K}$. If $g \in \mathcal{K}$, then $M g$ is equal to the zero game $g_{0}$ so $\|M g\|^{2}=\left\|g_{0}\right\|^{2}=0$ and the index is not well-defined. Notice that if $M g=g_{0}$, then $M g$ is both a zero-sum game and a common-interest game (in fact, $g_{0}$ it is the unique game that is both in $\mathcal{Z}$ and $\mathcal{C}$ ). We prefer to interpret it as being closer to a game without competitiveness so we set $\mathrm{CCI}(g)=0$ whenever $g \in \mathcal{K}$. This gives the index:

$$
\operatorname{CCI}(g)= \begin{cases}\frac{\|Z M g\|^{2}}{\|M g\|^{2}} & \text { if } g \in \mathcal{G} \backslash \mathcal{K}, \\ 0 & \text { if } g \in \mathcal{K} .\end{cases}
$$

This index is naturally bounded between 0 and 1 . If $g \neq g_{0}$ is a zero-sum game then $g=Z g$ and by Lemma 1:

$$
M g=M Z g=Z M g,
$$

so in this case $\operatorname{CCI}(g)=1$. If $g$ is a common-interest game then $g=C g$, so:

$$
M g=M C g=C M g=M g-Z M g,
$$

which means that $Z M g=g_{0}$, so $\mathrm{CCI}(g)=0$.

Statistical interpretation Our index also has a more statistical flavor. Using Lemma 1, we obtain:

$$
\begin{aligned}
g & =K g+M g, \\
& =K g+Z M g+C M g, \\
& =K g+M Z g+M C g, \\
& =K g+(Z g-K Z g)+(C g-K C g) .
\end{aligned}
$$

The first equality gives the decomposition of $g$ in its $\mathcal{M}$-part and its $\mathcal{K}$-part. The second equality decomposes the game $M g$ into its zero-sum component and its common-interest component. The third line uses the fact that $Z, M$ and $C, M$ commute. Finally, the last 
part uses the identities $Z g=M Z g+K Z g$ and $C g=M C g+K C g$. From this, we obtain the identity:

$$
g-K g=(Z g-K Z g)+(C g-K C g)
$$

Taking norms and recognizing that $Z g-K Z g=Z g-Z K g \in \mathcal{Z}$ and $C g-K C g=$ $C g-C K g \in \mathcal{C}$, we obtain that by orthogonality of $\mathcal{Z}$ and $\mathcal{C}$ :

$$
\frac{\|g-K g\|^{2}}{N|S|}=\frac{\|Z g-K Z g\|^{2}}{N|S|}+\frac{\|C g-K C g\|^{2}}{N|S|} .
$$

For $g=\left\{\pi_{i}: i \leq N\right\}$, the left hand side of Eq. (2) is equal to:

$$
\frac{1}{N} \sum_{i \leq N}\left[\frac{1}{|S|} \sum_{s \in S}\left(\pi_{i}(s)-\sum_{s^{\prime} \in S} \frac{1}{|S|} \pi_{i}\left(s^{\prime}\right)\right)^{2}\right] .
$$

This is the average of the variances of the players' payoff functions. Similarly, the first term on the right hand side of Eq. (2) gives the average of the variances of the payoff functions of the zero-sum part $Z g$ while the second term gives the average of the variances of the payoff functions of the common-interest game $C g$. If $g \neq K g$, then our index is given by

$$
\frac{\|Z M g\|^{2}}{\|M g\|^{2}}=\frac{\|Z(g-K g)\|^{2}}{\|g-K g\|^{2}}=\frac{\|Z g-K Z g\|^{2}}{\|g-K g\|^{2}} .
$$

The first equality uses the decomposition $g=M g+K g$ while the second equality uses the commutativity of $K$ and $Z$. The right hand side of Eq. (3) equals the first term on the right hand side of Eq. (2) divided by the left hand side of Eq. (2). This shows that we can also interpret our index as the fraction of the variances of the payoff functions in $g$ that is explained by the variances of the payoff-functions of the zero-sum part $Z g$. In other words, it shows how much of the variation in $g$ is be captured by the zero-sum game $Z g$. This is similar to an $R^{2}$-statistic in an OLS regression.

\section{Games with finite strategy sets}

In this section, we consider games with 2 players where each player has a finite number of (pure) strategies. Such games can be represented by two payoff matrices, say $A$ and $B$ that capture the payoff functions of the two players (see also Section 2 for an example). Here the element $a_{k, \ell}$ on row $k$ and column $l$ of matrix $A$ gives the payoff of player 1 if she plays her $k$-th strategy and player 2 chooses his $\ell$-th strategy. The same holds for the payoffs of player 2 and the matrix $B$. For two bimatrix games $g=\left\{\pi_{1}, \pi_{2}\right\}=(A, B)$ and $\tilde{g}=\left\{\tilde{\pi}_{1}, \tilde{\pi}_{2}\right\}=(\tilde{A}, \tilde{B})$, the inner product as defined in Eq. (1) takes the form:

$$
\begin{aligned}
\langle g, \tilde{g}\rangle & =\sum_{s \in S}\left(\pi_{1}(s) \tilde{\pi}_{1}(s)+\pi_{2}(s) \tilde{\pi}_{2}(s)\right) \\
& =\langle A, \tilde{A}\rangle+\langle B, \tilde{B}\rangle
\end{aligned}
$$


where $\langle A, \tilde{A}\rangle$ is the Frobenius inner product: $\langle A, \tilde{A}\rangle=\operatorname{trace}\left(A^{\prime} \tilde{A}\right)$.

Given a bimatrix game $g=(A, B)$, it is easily verified that the payoff matrices for $Z g$ are given by $(A-B) / 2$ and $(B-A) / 2$, while $K g$ is the game with payoff matrices $\bar{A}$ and $\bar{B}$, where $\bar{A}$ is the payoff matrix obtained from $A$ by substituting every element in $A$ by its average value (and similarly for $\bar{B}$ ). Straightforward computation then gives:

$$
\operatorname{CCI}(g)=\frac{\|(A-\bar{A})-(B-\bar{B})\|^{2}}{2\left(\|A-\bar{A}\|^{2}+\|B-\bar{B}\|^{2}\right)},
$$

where $\|A\|^{2}$ is the usual Frobenius norm $\|A\|^{2}=\operatorname{trace}\left(A^{\prime} A\right)$.

In the remaining part of this section, we will further investigate four classes of games that are popular in the literature: Prisoner's Dilemma games, Battle of the Sexes games, and games with strategic complements and substitutes.

\subsection{Prisoner's Dilemma}

A Prisoner's Dilemma game can be written as a symmetric $2 \times 2$ game:

\begin{tabular}{c|cc} 
& $\mathrm{C}$ & $\mathrm{D}$ \\
\hline $\mathrm{C}$ & $b, b$ & $d, e$ \\
$\mathrm{D}$ & $e, d$ & $f, f$
\end{tabular}

The game is a Prisoner's Dilemma if the payoffs are such that $e>b>f>d$. Moreover, let $2 b>d+e$ such that $(\mathrm{C}, \mathrm{C})$ both Pareto dominates the outcome $(\mathrm{D}, \mathrm{D})$ and maximizes joint payoffs. This game is one of the most frequently studied games in experimental economics. Usually, more cooperation is equated with a higher frequency of playing $(\mathrm{C}, \mathrm{C})$. We first derive the CCI for this game. Next, we present two seminal recent experiments to illustrate our index. Finally, we proceed by providing a more complete picture of the literature.

Derivation of the CCI A first step to obtain our index is to compute the average payoff for each player: $\bar{\pi}_{1}=\bar{\pi}_{2}=\frac{b+d+e+f}{4}$. Given this, the mean-normalized game $(A-\bar{A}, B-\bar{B})$ is given by:

\begin{tabular}{c|cc} 
& $\mathrm{C}$ & $\mathrm{D}$ \\
\hline $\mathrm{C}$ & $\frac{3 b-d-e-f}{4}, \frac{3 b-d-e-f}{4}$ & $\frac{3 d-b-e-f}{4}, \frac{3 e-b-d-f}{4}$ \\
$\mathrm{D}$ & $\frac{3 e-b-d-f}{4}, \frac{3 d-b-e-f}{4}$ & $\frac{3 f-b-d-e}{4}, \frac{3 f-b-d-e}{4}$
\end{tabular}

After some tedious but straightforward calculations, we obtain:

$$
\begin{aligned}
\mathrm{CCI} & =\frac{\|(A-\bar{A})-(B-\bar{B})\|^{2}}{2\left(\|A-\bar{A}\|^{2}+\|B-\bar{B}\|^{2}\right)}, \\
& =\frac{2(d-e)^{2}}{(b-d)^{2}+(b-e)^{2}+(b-f)^{2}+(d-e)^{2}+(d-f)^{2}+(e-f)^{2}} .
\end{aligned}
$$


When interests are perfectly aligned, i.e., $d=e$, we obtain the value $\operatorname{CCI}(g)=0$. Next, we can consider comparative statics in the various parameters. For a marginal change in $b$ - the payoff at cooperation - it suffices to look at the derivative of the denominator:

$$
\frac{\partial d e n o m(\mathrm{CCI})}{\partial b}=2(b-d)+2(b-e)+2(b-f) .
$$

The index of competitiveness increases in $b$ when $b$ is below the average payoff and decreases in $b$ when $b$ is above the average payoff, which is the case in the Prisoner's Dilemma game. A similar calculation reveals the same comparative static in $f$, i.e. for the Prisoner's Dilemma game, if $f$ is below the average payoff, then the index increases in $f$. The comparative statics in $d$ and $e$ are more difficult to interpret as they might change for different values of the parameters. However, consider following reparametrization:

$$
\begin{aligned}
& \Delta=e-d, \\
& \Gamma=e+d .
\end{aligned}
$$

Here $\Delta$ measures the payoff difference in the off-diagonal elements while $\Gamma$ measures the sum of the off-diagonal payoff. Substituting $d=(\Gamma-\Delta) / 2$ and $e=(\Gamma+\Delta) / 2$ into above expression for the CCI, one finds (after some careful calculations) that the CCI is increasing in $\Delta$ (for fixed $\Gamma$ ). Also, the CCI is increasing in $\Gamma$ (for fixed $\Delta$ ) as long as $e+d<b+f$.

Connections to the experimental literature We first illustrate our approach with two seminal recent experiments. Friedman and Oprea (2012) conducted a (quasi-)continuous time experiment in which subjects can adapt strategies in real time. They consider four parametrizations in which $b=10, d=0, e \in\{14,18\}$ and $f \in\{4,8\}$. Calculations show that:

$$
\mathrm{CCI}_{14,4}=0.844<\mathrm{CCI}_{18,4}=0.880<\mathrm{CCI}_{14,8}=0.942<\mathrm{CCI}_{18,8}=0.987
$$

Next, Dal Bó and Fréchette (2011) consider an infinitely repeated Prisoner's Dilemma game. They use the parameters $b \in\{32,40,48\}, d=12, e=50, f=25$. Calculations show that

$$
\mathrm{CCI}_{32}=0.959>\mathrm{CCI}_{40}=0.863>\mathrm{CCI}_{48}=0.710 .
$$

In both of the above experiments, the ordering of the CCI matches the observed level of cooperation. To provide a more comprehensive comparison, we compute our index to the data of 96 other experiments taken from 24 studies on the Prisoner's Dilemma game from a meta-study by Mengel (2017). We compare our index to other indices which are used to explain cooperation rates. Mengel (2017) employs three indices: (i) the temptation to defect, measured by the percentage gain when unilaterally defecting against a cooperator (TEMPT), (ii) the risk to cooperate, measured by the percentage loss of unilaterally cooperating against a defector (RISK), and (iii) the efficiency, measured by the gain from 
mutual cooperation compared to the payoff obtained under mutual defection (EFF). ${ }^{7}$ The conclusion of Mengel (2017) is that RISK provides the best predictor for cooperation across one-shot games, while TEMPT plays a more important role in finitely repeated games. Recently, Embrey, Fréchette, and Yuksel (2018) use an index called 'Basin of Attraction of Defection' $(\mathrm{BAD})^{8}$ to explain cooperation in finitely repeated Prisoner's Dilemma games. They assume that players will decide only between two strategies: the grim-trigger strategy (cooperate first and defect until the end of the game as soon as the opponent defects once) and the always-defect strategy. BAD is the probability that a player must assign to the other player playing the grim-trigger strategy so that he is indifferent between playing grim-trigger and always-defect. The higher value of BAD suggests less support for cooperation because a player must have a higher belief that her partner is a conditional cooperator.

We compare the explanatory power of our index with these indices, with and without controlling for the matching protocol (stranger vs partner matching) and study fixedeffects. The OLS estimates are reported in Table 1. In all cases, our index delivers strong negative correlations and explanatory power (in terms of the $\mathrm{R}^{2}$ ) comparable to the other indices.

When judging the comparisons between these indices and the CCI, one should bear in mind that these other indices are specifically constructed for the Prisoner's Dilemma. For example, for a linear Public Goods game, it is not possible to create the same variation in RISK and TEMPT as both variables co-move with the same parameter (i.e., the marginal per capita rate of return; see Section 5.2 below). Our index has the versatility that it can also be applied to a wide variety of other games.

\subsection{Battle of the Sexes}

We now consider the Battle of the Sexes game. This game presents a situation where two players prefer to agree on a common strategy to play, but their preferences diverge over which common strategy is the best. Introducing two parameters $s \geq t>0$, the Battle of the Sexes has the following structure:

\begin{tabular}{c|cc} 
& $\mathrm{X}$ & $\mathrm{Y}$ \\
\hline $\mathrm{X}$ & $s, t$ & 0,0 \\
$\mathrm{Y}$ & 0,0 & $t, s$
\end{tabular}

Both players agree that $(X, X)$ and $(Y, Y)$ are better than the off diagonal outcomes but when $s>t$ then player 1 prefers $(X, X)$ over $(Y, Y)$ while player 2 prefers $(Y, Y)$ over $(X, X)$. In order to compute the CCI, we first compute the average payoff for each player which is here equal to $\frac{s+t}{4}$. Thus, the normalized game $(A-\bar{A}, B-\bar{B})$ is:

\footnotetext{
${ }^{7}$ Formally, TEMPT $=(e-b) / e, \operatorname{RISK}=(f-d) / f$ and $\operatorname{EFF}=(b-f) / b$. If $0 \geq f>d$, RISK $\equiv$ $(|f-d|) /|d|$ so that RISK $\in[0 ; 1]$. When $b<0<f$, EFF $\equiv 1$ to avoid non-monotonicity, as EFF $=1$ if $f=0$. When $d=0$, a noise term drawn uniformly form the open unit interval is added to $d$. In the regression results reported in Table 1 we use the real experimental values of $d$.

${ }^{8} \mathrm{BAD}=(-d+f) /((b-f) T-d-e+2 f)$, where $T$ is the number of rounds in the repeated game.
} 
Table 1: Average cooperation rates in Prisoner's Dilemma regressed on variables of interest (data from Mengel, 2017)

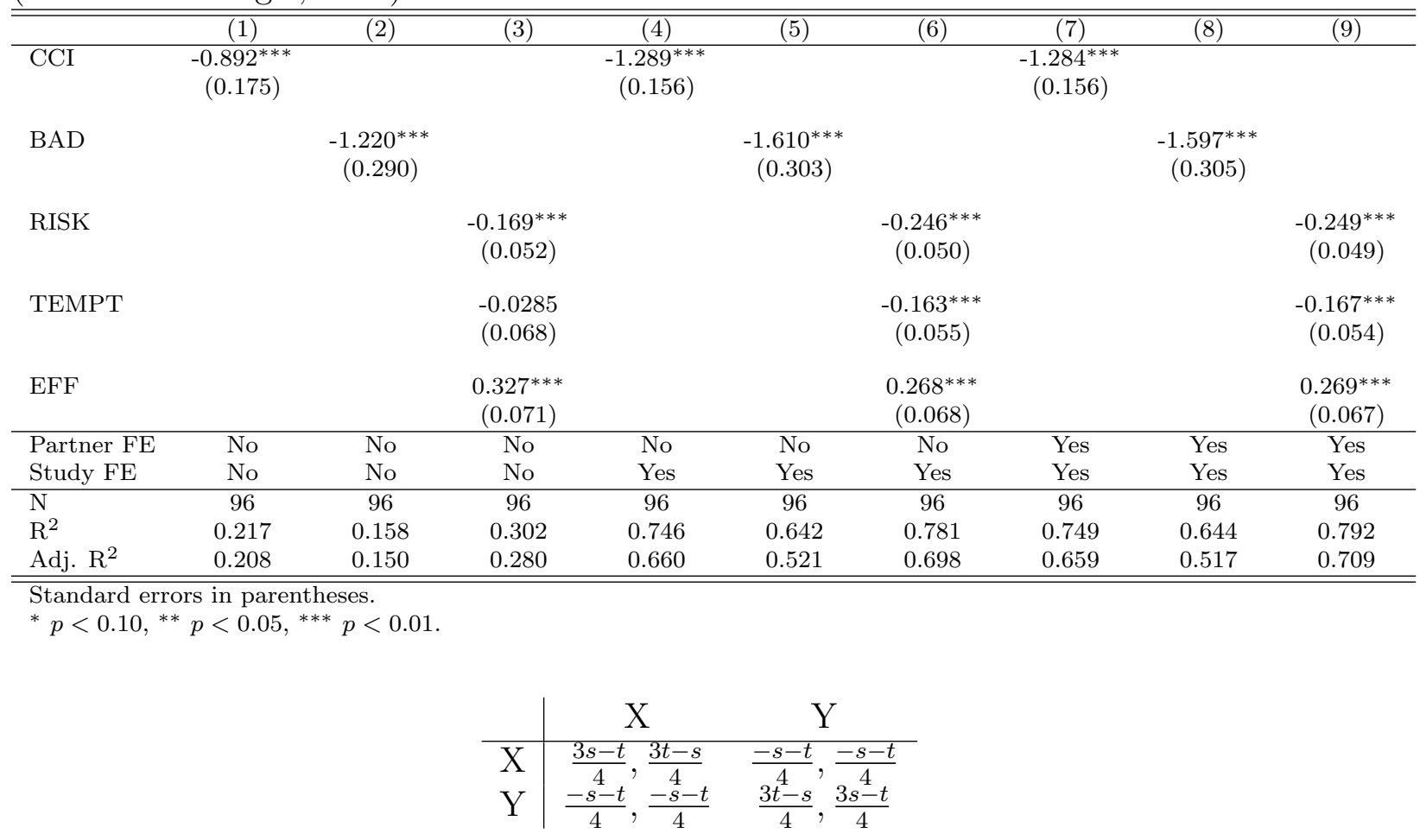

For our index, we obtain:

$$
\begin{aligned}
\mathrm{CCI} & =\frac{\|(A-\bar{A})-\left(B-\bar{B} \|^{2}\right.}{2\left(\|A-\bar{A}\|^{2}+\|B-\bar{B}\|^{2}\right)}=\frac{2(s-t)^{2}}{2\left(2\left(\left(\frac{3 s-t}{4}\right)^{2}+2\left(\frac{s+t}{4}\right)^{2}+\left(\frac{3 t-s}{4}\right)^{2}\right)\right)}, \\
& =\frac{8(s-t)^{2}}{(3 s-t)^{2}+2(s+t)^{2}+(3 t-s)^{2}} .
\end{aligned}
$$

If the game is a pure coordination game, i.e., $s=t$, we obtain CCI $=0$. For an increase in $s$, we have $\frac{\partial \mathrm{CCI}}{\partial s}=\frac{8 t\left(s^{2}-t^{2}\right)}{\left(3 s^{2}-2 s t+3 t^{2}\right)^{2}}$. If $s>t>0$, both numerator (and denominator) are positive, i.e., the game becomes more competitive. The reverse comparative statics hold for changes in $t$. Thus, our index agrees with the intuition that it becomes harder to coordinate/cooperate when the payoffs on the main diagonal are less aligned.

We now contrast our index with experimental results in this coordination game. There are a limited number of studies that allow for variations in $s$ or $t$ (and also allow for $s$ and $t$ to be distinct). Both Crawford, Gneezy, and Rottenstreich (2008) and Parravano and Poulsen (2015) consider an experimental implementation in a one-shot game for different values of $s$ and $t$. They measure the percentage of successful coordination. In the symmetric treatments $(s=t)$, they document the highest percentage. For both small and large asymmetries in payoffs, the extent of coordination is comparable.

A critical reader might argue that in a one-shot interaction, the coordination problem hinders cooperation. As such, the fraction of $(\mathrm{X}, \mathrm{X})$ and $(\mathrm{Y}, \mathrm{Y})$ outcomes might be low even 
though both players would like to coordinate on one of these outcomes. To shed some light on this, Elten and Penczynski (2018) allow for intra-group communication. In particular, each member in a group of two can send a message consisting of a suggested decision that represents a subject's proposal for the joint group action. Then, the other side receives the message and can make a final decision. They find that the coordination rates increase for treatments with $s=t$, but are comparable to those without communication for the asymmetric treatments.

Of course, even with this communication protocol, there is still room for miscoordination. To abstract from coordination problems in order to focus on cooperation, it would thus be interesting to implement a one-shot version with more natural communication protocol, e.g., sending messages back and forth. Alternatively, one could implement a repeated version of the game with partner matching to see whether, after some initial rounds, expected coordination rates come close to a fully cooperative outcome even when payoffs are not aligned.

\subsection{Games of Strategic Complements and Substitutes}

A literature where notions of competitiveness or cooperativeness (collusion) are prevalent is industrial organization. Within this literature, there has been a large focus on the study of games with strategic complements and games with strategic substitutes. A game has strategic complements if a change in a player's strategy gives an incentive for the other player to move in the same direction. Under strategic substitutes, the incentive is to move in the opposite direction. A classroom example of a game with strategic complements is an oligopoly with price competition where goods are substitutes. Oligopoly models with quantity competition usually have strategic substitutes.

A seminal contribution to the experimental literature on games with strategic substitutes and complements is Potters and Suetens (2009). Their experiment uses four carefully designed treatments: (i) strategic substitutes with negative externalities (SUBSTneg), (ii) strategic substitutes with positive externalities (SUBSTpos), (iii) strategic complements with negative externalities (COMPLneg), and (iv) strategic complements with positive externalities (COMPLpos). Their experimental design has several nice features. Most importantly for us, all four treatments have the same strategy sets. This allows us to compute and compare the CCI over the various treatments. ${ }^{9}$ In addition, all four treatments have the same choices and payoffs for the Nash equilibrium, the same choices (conditional on the sign of the externality) and the same payoffs for the joint payoff maximizing outcome, the same optimal defection payoffs, and the same best-response functions' slopes (in absolute

\footnotetext{
${ }^{9}$ Although there exists a vast number of other experiments in the literature that focus on the classical models of industrial organization, most of them focus on treatment variations for which the CCI index can not be used to conduct comparative statics. In particular, many studies change the matching or feedback protocol, the strategy space, or the number of players over the treatments. On the other hand, as is clear from the theoretical framework developed in Section 3, the CCI index should only be applied to compare settings where the space of strategies and the number of players are fixed. Given this, we advise not to use the CCI to compare games that vary in the number of players or the strategy space.
} 
values).

Potters and Suetens (2009) define the degree of cooperation, as the ratio between (i) the difference between the average observed choices and the choice at the Nash equilibrium, and (ii) the difference between the joint payoff-maximizing choice and the choice at the Nash equilibrium. ${ }^{10}$ Their experimental design matches subjects in pairs. Each pair plays one of the four treatments 31 times. Every treatment has between 13 and 14 independent observations (total number of subjects is 110).

Their first main result is that the sign of the externality does not have a (statistically) significant effect on the degree of cooperation. This is supported by our CCI index which is the same for SUBSTneg and SUBSTpos and the same for COMPLneg and COMPLpos.

On the other hand, as the results show, they do find that the degree of cooperation is larger for games of strategic complements compared to the games with strategic substitutes. Again, the values of the CCI are in line with this result. Specifically, the CCI equals 0.4271 in both treatments with strategic complements, while the CCI is equal to 0.5033 in for both treatments with strategic substitutes.

\section{$5 \quad$ Games with infinite strategy sets}

We now turn to games in which each player has an infinite, continuous set of strategies. In all of our examples, we can follow the simple intuition of replacing summation by integration when defining the inner product. Formally, for games $g=\left\{\pi_{i}: i \leq N\right\}$ and $\tilde{g}=\{\tilde{\pi}: i \leq N\} \in \mathcal{G}$, we can define an inner product $\langle.,$.$\rangle on \mathcal{G}$ by

$$
\langle g, \tilde{g}\rangle=\sum_{i=1}^{N} \int_{S} \pi_{i}(s) \tilde{\pi}_{i}(s) Q(d s) \equiv \sum_{i=1}^{N} \int_{S} \pi_{i} \tilde{\pi}_{i} d Q,
$$

where $Q$ is a measure on the set of strategy profiles $S$. We will take $Q$ to be the Lebesgue measure throughout this section. A more precise and general derivation for the infinite strategy setting is presented in Appendix A.

\subsection{Contests}

As a first application in this section, we look at three different contest models. We first compare two of the most commonly used contest models, the All-pay auction and the Tullock contest. Finally, we consider a contest in which an endowment can be shared between productive and fighting activities. For tractability and comparability, we focus on a simple version with two symmetric players.

All-pay auction We start our analysis with the All-pay auction. Consider two players $i=1,2$ with valuations $v_{1}=v_{2}=v$ for winning the auction. Each player chooses a

\footnotetext{
${ }^{10}$ Computing the degree of coopertion in terms of payoffs compared to choices does not alter their conclusions.
} 
bid/effort level $x_{i} \in[0, v]$. The player with the higher effort level wins. In case of a tie, the prize is shared. ${ }^{11}$ The payoff function of each player is thus given by:

$$
\pi_{i}\left(x_{i}, x_{j}\right)= \begin{cases}v-x_{i} & \text { if } x_{i}>x_{j} \\ \frac{v}{2}-x_{i} & \text { if } x_{i}=x_{j} \\ -x_{i} & \text { if } x_{i}<x_{j}\end{cases}
$$

The average payoff can thus be written as: ${ }^{12}$

$\bar{\pi}_{i}=\int_{0}^{v} \int_{0}^{v} \frac{1}{v} \frac{1}{v}\left(\mathbf{1}_{\left\{x_{i}>x_{j}\right\}}\left(v-x_{i}\right)+\mathbf{1}_{\left\{x_{i}<x_{j}\right\}}\left(-x_{i}\right)\right) \mathrm{d} x_{j} \mathrm{~d} x_{i}=\frac{1}{v^{2}} \int_{0}^{v} \int_{0}^{v}\left(\mathbf{1}_{\left\{x_{i}>x_{j}\right\}} v-x_{i}\right) \mathrm{d} x_{j} \mathrm{~d} x_{i}$.

Note that:

$$
\int_{0}^{v} \int_{0}^{v}\left(\mathbf{1}_{\left\{x_{i}>x_{j}\right\}} v-x_{i}\right) \mathrm{d} x_{j} \mathrm{~d} x_{i}=\int_{0}^{v} \int_{0}^{x_{i}} v \mathrm{~d} x_{j} \mathrm{~d} x_{i}-\int_{0}^{v} x_{i} v \mathrm{~d} x_{i}=0 .
$$

Thus, this game is already mean-normalized, i.e., $\bar{\pi}_{i}=0$. Let us calculate the terms needed for our index:

$$
\begin{gathered}
\left\|\pi_{1}\left(x_{1}, x_{2}\right)-\pi_{2}\left(x_{1}, x_{2}\right)\right\|^{2}=\int_{0}^{v} \int_{0}^{v} \frac{1}{v^{2}}\left(v\left(\mathbf{1}_{\left\{x_{1}>x_{2}\right\}}-\mathbf{1}_{\left\{x_{2}>x_{1}\right\}}\right)-x_{1}+x_{2}\right)^{2} \mathrm{~d} x_{2} \mathrm{~d} x_{1}, \\
=\frac{1}{v^{2}} \int_{0}^{v} \int_{0}^{v}\left(v-\left|x_{1}-x_{2}\right|\right)^{2} \mathrm{~d} x_{2} \mathrm{~d} x_{1}=\frac{v^{2}}{2} \\
\left\|\pi_{1}\left(x_{1}, x_{2}\right)\right\|^{2}=\left\|\pi_{2}\left(x_{1}, x_{2}\right)\right\|^{2}=\int_{0}^{v} \int_{0}^{v} \frac{1}{v^{2}}\left(\mathbf{1}_{\left\{x_{1}>x_{2}\right\}} v-x_{1}\right)^{2} \mathrm{~d} x_{2} \mathrm{~d} x_{1}=\frac{v^{2}}{6} .
\end{gathered}
$$

Hence, the CCI for the All-pay auction is:

$$
\mathrm{CCI}=\frac{\left\|\pi_{1}\left(x_{1}, x_{2}\right)-\pi_{2}\left(x_{1}, x_{2}\right)\right\|^{2}}{2\left(\left\|\pi_{1}\left(x_{1}, x_{2}\right)\right\|^{2}+\left\|\pi_{2}\left(x_{1}, x_{2}\right)\right\|^{2}\right)}=\frac{\frac{v^{2}}{2}}{2\left(\frac{v^{2}}{6}+\frac{v^{2}}{6}\right)}=\frac{3}{4}
$$

Tullock contest We compare the All-pay auction model with a Tullock contest. For this comparison, we keep the same two players $i=1,2$ with the same valuations $v_{1}=v_{2}=v$ for winning the prize. Again, each player chooses a bid/effort level $x_{i} \in[0, v]$. In this part, we consider a variant in which the player with the higher effort level gets a larger share of

\footnotetext{
${ }^{11}$ Ties are a measure zero event in the strategy space. Thus, our results are independent of the specification of the tie-breaking rule.

${ }^{12}$ In the following calculations of the double integrals for our index, we can freely change the order of integration by the Fubini-Tonelli Theorem, since all terms are either non-negative or integrable.
} 
the prize; see e.g., Cason, Masters and Sheremeta $(2010,2018) .{ }^{13}$ The payoff function of each player is given by:

$$
\pi_{i}\left(x_{i}, x_{j}\right)= \begin{cases}\frac{v}{2} & \text { if } x_{i}=x_{j}=0, \\ v \frac{x_{i}^{\alpha}}{x_{i}^{\alpha}+x_{j}^{\alpha}}-x_{i} & \text { otherwise }\end{cases}
$$

where $\alpha>0$ is the decisiveness parameter. As $\alpha \rightarrow \infty$, the Tullock contest converges to an All-pay auction.

For the most often analyzed Tullock lottery contest where $\alpha=1$, we obtain for the average payoff:

$$
\bar{\pi}_{i}=\int_{0}^{v} \int_{0}^{v} \frac{1}{v} \frac{1}{v}\left(\left(\frac{x_{i}}{x_{i}+x_{j}}\right) v-x_{i}\right) \mathrm{d} x_{j} \mathrm{~d} x_{i}=0 .
$$

Thus, this game is also mean-normalized, i.e., $\bar{\pi}_{i}=0$. The calculation of our index yields:

$$
\mathrm{CCI}=\frac{\left\|\pi_{1}\left(x_{1}, x_{2}\right)-\pi_{2}\left(x_{1}, x_{2}\right)\right\|^{2}}{2\left(\left\|\pi_{1}\left(x_{1}, x_{2}\right)\right\|^{2}+\left\|\pi_{2}\left(x_{1}, x_{2}\right)\right\|^{2}\right)}=0.155 .
$$

According to our index, the Tullock lottery contest with sharing is much less competitive than the All-pay auction. This is consistent with the common view that contests are more competitive when they are more decisive.

In Appendix B, we derive our competitiveness-cooperativeness index for $\alpha=3$ and obtain CCI $=0.48$. Millner and Pratt (1989) provide an experimental comparison between the cases $\alpha=1$ and $\alpha=3 .^{14}$ Consistent with our index, they report higher (socially wasteful) contest expenditures when $\alpha=3$, which reflects a more competitive environment.

The contest model of Durham, Hirshleifer, and Smith As a third contest model, we consider the model of Durham, Hirshleifer, and Smith (1998). Again, we restrict attention to the most simplified 2 player version, where each player is endowed with an amount $v$. Each player $i$ decides how to split the endowment $v$ between productive effort $x_{i}$ and fighting effort $v-x_{i}$. The payoff function of each player $i$ is given by:

$$
\pi_{i}=\frac{x_{i}^{r}}{x_{i}^{r}+x_{j}^{r}}\left(v-x_{i}\right)\left(v-x_{j}\right) .
$$

The symmetric treatments in the experiment of Durham, Hirshleifer, and Smith (1998) use the values $v=20$ and $r \in\{1,4\}$. In Appendix B, we show that for $r=1$ and $v=20$, we have CCI $=0.236$ and for $r=4$ and $v=20$, we have CCI $=0.433$. Thus, the game with $r=1$ is predicted to be less competitive. The experimental implementation of Durham, Hirshleifer and Smith (1998) uses different matching protocols. For all of them, the shares

\footnotetext{
${ }^{13}$ In the classical lottery contest, a higher effort leads to a higher winning probability instead of a larger share. The expected payoffs in the two games are equivalent. For the lottery case, it seems natural to use expected payoffs for our index of competitiveness. In this case, the analysis in this section also holds for the probabilistic Tullock contest.

${ }^{14}$ Millner and Pratt (1989) use the probabilistic version of the Tullock contest.
} 
of the endowment that is spend on fighting are higher for $r=4$ compared to $r=1$ and the resulting payoffs are lower for $r=4$ compared to $r=1$. Again, this matches the prediction of our index that the game with $r=4$ is more competitive.

\subsection{Public Goods game}

In a final application, we demonstrate how our index can be applied to games with more than 2 players. For this purpose, we use the well-known Public Goods game. We consider a setting where each player has a unit endowment and decides to invest a fraction $1-x_{i} \in$ $[0,1]$ in the production of a public good and keep the rest to herself. The investment into the public good gives a return of $\alpha\left(1-x_{i}\right)$ to all players. As such, the payoff of player $i$ is given by:

$$
\pi_{i}\left(x_{1}, \ldots, x_{N}\right)=x_{i}+\alpha \sum_{j}\left(1-x_{j}\right) .
$$

The average payoff of player $i$ is then:

$$
\begin{aligned}
\bar{\pi}_{i}= & \int_{0}^{1} \ldots \int_{0}^{1}\left(x_{i}+\alpha \sum_{j=1}^{N}\left(1-x_{j}\right)\right) \mathrm{d} x_{N} \ldots \mathrm{d} x_{1}, \\
& =\frac{1}{2}+\frac{\alpha N}{2} .
\end{aligned}
$$

As such, the normalized payoff is given by:

$$
\pi_{i}-\bar{\pi}_{i}=x_{i}-\alpha \sum_{j=1}^{N} x_{j}+\frac{\alpha N-1}{2} .
$$

After some calculations (see Appendix B), we obtain:

$$
\mathrm{CCI}=\frac{(N-1)}{(\alpha N-1)^{2}+(N-1)} .
$$

For $\alpha=1 / N$ the index equals CCI $=1$. For $\alpha>1 / N$ the index is less than 1 and is decreasing in $\alpha$.

Concerning experimental evidence, we can look at public goods provision when $\alpha$ changes, keeping all other factors fixed (e.g., number of players). In a meta-analysis of 27 studies, Zelmer (2003) reports that the marginal per capita return $\alpha$ has a significant positive effect on the average level of contribution to the public goods game. Since the CCI is decreasing in $\alpha$, this result is in line with the predictions of our index.

\section{Discussion}

We introduced an index of competitiveness and cooperativeness, the CCI, that can be computed using only the primitives of the game. In our applications, we have used the 
index as a predictor for cooperative or competitive behavior. Within each class of games, we have shown that the CCI correlates with empirical findings from the literature.

We would like to emphasize that the CCI can be used both for settings that study competitiveness as well as for settings that focus on cooperativeness. In this sense, our index can be seen as a unifying framework that has pure cooperativeness and pure competitiveness as the extremes. Relatedly, given the theoretical framework, we have taken a conservative approach and we have not compared our measure across games with different strategy spaces. Extending our measure to take into account varying number of players and changing strategy sets is an obvious area for future research.

Since our index is widely applicable, we hope to inspire more experimental research on the effects of competitiveness for different games. Two natural starting points would be to look at changes in behavior in future rounds after playing a more competitive game and gender differences in the selection into more competitive games.

Finally, we want to emphasize that our index should be seen as one component in predicting competition or cooperation. In this sense, it is complimentary to other approaches which study the importance of procedural aspects such as the matching protocol and the number of rounds in an experiment. A potential avenue for experimental research might be to combine both approaches. For instance, one could study how much the protocol has to change (e.g., increase the number of rounds, use partner rather than stranger matching) to restore a certain level of cooperation when a game becomes more competitive.

\section{References}

Buser, T. And A. Dreber (2016), "The Flipside of Comparative Payment Schemes" Management Science, 62, 2626-2638.

Candogan, O, Menache, I., Ozdaglar, A., and P.A. Parrilo (2011), "Flows and Decompositions of Games: Harmonic and Potential Games", Mathematics of Operations Research, 36, 474-503.

Carpenter, J. AND E. SEKi (2006), "Competitive work environments and social preferences: Field experimental evidence from a japanese fishing community", Contributions to Economic Analysis $\mathcal{G}$ Policy, BE Press, 5, Article 2.

Cason, T.N., Masters, W.A., And R.M. Sheremeta (2010), "Entry into winnertake-all and proportional-prize contests: An experimental study", Journal of Public Economics, 94, 604-611.

Cason, T.N., Masters, W.A., and R.M. Sheremeta (2018), "Winner-Take-All and Proportional Prize Contests: Theory and Experimental Results, Journal of Economic Behavior and Organization", forthcoming 
Crawford, V.P., Gneezy, U., And Y. Rottenstreich (2008), "The Power of Focal Points Is Limited: Even Minute Payoff Asymmetry May Yield Large Coordination Failures", American Economic Review, 98, 1443-1458.

Dal Bó, P. and G. Fréchette (2011), "The Evolution of Cooperation in Infinitely Repeated Games: Experimental Evidence", American Economic Review, 100, 22052229 .

Durham, Y., Hirshleifer, J., And V. L. Smith (1998), "Do the Rich Get Richer and the Poor Poorer? Experimental Tests of a Model of Power", American Economic Review, 88, 970-983.

Embrey, M., Fréchette, G.R. And S. Yuksel (2018), "Cooperation in the Finitely Repeated Prisoner's Dilemma", Quarterly Journal of Economics, 133, 509-551.

Elten, J., And S. P. Penczynski (2018), "Coordination games with asymmetric payoffs: An experimental study with intra-group communication", Working paper.

Facchini, G., van Megen, F., Borm, P., And S. Tijs (1997), "Congestion models and weighted Bayesian potential games", Theory and Decision, 42, 193-206.

Friedman, D. and R. Oprea (2012), "A Continuous Dilemma", American Economic Review, 102, 337-363.

Gneezy, U., Niederle M. And A. Rustichini (2003), "Performance in Competitive Environments: Gender Differences", Quarterly Journal of Economics, 118, 10491074.

Harsanyi, R. C. and R. Selten (1988), A General Theory of Equilibrium Selection in Games, The MIT Press, Cambridge.

Kalai, A. And Kalai, E. (2013), "Cooperation in strategic games revisited", Quarterly Journal of Economics, 128, 917-966.

Mengel, F. (2017), "Risk and Temptation: A Meta-study on Prisoner's Dilemma Games", Economic Journal, 128, 3182-3209.

Millner, E.L. And M.D. Pratt (1989), "An experimental investigation of efficient rent-seeking", Public Choice, 62, 139-151.

Parravano, M. And O. Poulsen (2015), "Stake size and the power of focal points in coordination games: Experimental Evidence", Games and Economic Behavior, 94, 191-199.

Peysakhovich, A. and D. G. Rand (2015), "Habits of virtue: Creating norms of cooperation and defection in the laboratory", Management Science, 62, 631-647. 
Potters, J. And S. Suetens (2009), "Cooperation in Experimental Games of Strategic Complements and Substitutes", Review of Economic Studies, 76(3), 1125-1147.

Roth, A.E., And J.K. Murnighan (1978), "Equilibrium Behavior and Repeated Play of the Prisoner's Dilemma" Journal of Mathematical Psychology, 17, 189-198.

Weibull, J. (1995), Evolutionary Game Theory, The MIT Press, Cambridge.

Zelmer, J. (2003), "Linear Public Goods Experiments: A Meta-Analysis" Experimental Economics, 6, 299-310. 


\section{Appendix A: Derivation for general continuous games and proofs of Lemma 1 and Proposition 1}

Consider a finite set of $N$ players and for every player a set of strategies $S_{i}$. Unlike the main text, we also allow the sets $S_{i}$ to be infinite. Let $S=\prod_{i \leq N} S_{i}$ be the set of strategy profiles. Let $\mathcal{S}$ be a $\sigma$-algebra on $S$ such that $(S, \mathcal{S})$ is a measurable space. In addition, let $Q$ be a measure on $(S, \mathcal{S})$.

Let $\mathcal{G}$ be the set of games $g=\left\{\pi_{i}: i \leq N\right\}$ such that for all $i \leq N, \pi_{i} \in L_{2}(Q) .{ }^{15}$ For $g=\left\{\pi_{i}: i \leq N\right\}, h=\{\tilde{\pi}: i \leq N\} \in \mathcal{G}$, we define the following inner product $\langle.,$.$\rangle on \mathcal{G}$ :

$$
\langle g, h\rangle=\sum_{i=1}^{N} \int_{S} \pi_{i}(s) \tilde{\pi}_{i}(s) Q(d s) \equiv \sum_{i=1}^{N} \int_{S} \pi_{i} \tilde{\pi}_{i} d Q .
$$

By varying the measure $Q$ we can give more or less weight to certain strategies. For the applications in the main part of the paper, we used the Lebesgue measure for $Q$.

Observe that the norm $\|g\|=\sqrt{\langle g, g\rangle}$ treats two games in the same way if their payoff functions are equal $Q$-a.s.. In other words, in order to be technically correct, we should define the set of games $\mathcal{G}$ only on the partition induced by the measure $Q$. For ease of notation, however, we omit this from the notation, but keep in mind that all statements in the appendix only hold $Q$-a.s..

Compared to the main text, the sets $\mathcal{K}, \mathcal{M}, \mathcal{Z}$ and $\mathcal{C}$ differ marginally,

$$
\begin{aligned}
& \mathcal{K}=\left\{g=\left\{\pi_{i}: i \leq N\right\} \in \mathcal{G}: \forall i \leq N, \pi_{i} \text { is constant }\right\}, \\
& \mathcal{M}=\left\{g=\left\{\pi_{i}: i \leq N\right\} \in \mathcal{G}: \forall i \leq N \int_{S} \pi_{i} d Q=0\right\}, \\
& \mathcal{Z}=\left\{g=\left\{\pi_{i}: i \leq N\right\} \in \mathcal{G}: \forall s \in S, \sum_{i \leq N} \pi_{i}=0\right\}, \\
& \mathcal{C}=\left\{g=\left\{\pi_{i}: i \leq N\right\} \in \mathcal{G}: \forall i, j \leq N, \pi_{i}=\pi_{j}\right\} .
\end{aligned}
$$

The proof the following lemma includes the proof Lemma 1 in the main text.

\section{Lemma 2.}

1. $\mathcal{M}^{\perp}=\mathcal{K}$ and $\mathcal{Z}^{\perp}=\mathcal{C}$.

2. Every game g can be uniquely decomposed as

$$
g=\hat{g}+\tilde{g}
$$

where $\hat{g} \in \mathcal{M}$ and $\tilde{g} \in \mathcal{K}$. In particular $\hat{g}=M g$ and $\tilde{g}=K g$. Moreover, in this case,

$$
\|g\|^{2}=\|\hat{g}\|^{2}+\|\tilde{g}\|^{2} .
$$

\footnotetext{
${ }^{15}$ Here $L_{2}(Q)$ is the set of $Q$-measurable functions $\pi_{i}: S \rightarrow \mathbb{R}$ such that $\int_{S}\left(\pi_{i}\right)^{2} d Q<\infty$.
} 
3. Every game g can be uniquely decomposed as,

$$
g=\hat{g}+\tilde{g}
$$

where $\hat{g} \in \mathcal{Z}$ and $\tilde{g} \in \mathcal{C}$. In particular $\hat{g}=Z g$ and $\tilde{g}=C g$. Moreover, in this case,

$$
\|g\|^{2}=\|\hat{g}\|^{2}+\|\tilde{g}\|^{2} .
$$

4. For $g=\left\{\pi_{i}: i \leq N\right\}$ we have that

- $K g=\left\{\tilde{\pi}_{i}: i \leq N\right\}$ is such that, $\tilde{\pi}_{i}=\frac{\int_{S} \pi_{i} d Q}{\int_{S} d Q}$.

- $C g=\left\{\tilde{\pi}_{i}: i \leq N\right\}$ is such that, $\tilde{\pi}_{i}=\frac{\sum_{j \leq N} \pi_{j}}{N}$.

5. The operators $(M, Z),(M, C),(K, Z)$ and $(K, C)$ are commutative. In other words, for all $g \in \mathcal{G}, M Z g=Z M g, K Z g=Z K g, M C g=C M g$ and $K C g=C K g$.

Proof. Part 1: We will start by showing the equivalence $\mathcal{K}^{\perp}=\mathcal{M}$. Let $\tilde{g}=\{\tilde{\pi}: i \leq N\} \in$ $\mathcal{K}^{\perp}$. Then, for all $g=\left\{\pi_{i}: i \leq N\right\} \in \mathcal{K}$,

$$
\langle\tilde{g}, g\rangle=0=\sum_{i \leq N} \int_{S} \pi_{i} \tilde{\pi}_{i} d Q .
$$

Let $g$ be such that for $i \leq N, \pi_{i}=1 \in \mathbb{R}$ and for $j \neq i, \pi_{j}=0$. Then,

$$
0=\int_{S} \pi_{i} d Q
$$

which shows that $\tilde{g} \in \mathcal{M}$.

Also, if $\tilde{g}=\left\{\tilde{\pi}_{i}: i \leq N\right\} \in \mathcal{M}$ and $g=\left\{\pi_{i}: i \leq N\right\} \in \mathcal{K}$ with $\pi_{i}=c_{i} \in \mathbb{R}$, then,

$$
\langle\tilde{g}, g\rangle=\sum_{i \leq N} \int_{S} \pi \tilde{\pi} d Q=\sum_{i \leq N} c_{i} \int_{S} \tilde{\pi}_{i} d Q=0 .
$$

Next, we will show that $\mathcal{C}=\mathcal{Z}^{\perp}$. Let $\tilde{g}=\{\tilde{\pi}: i \leq N\} \in \mathcal{Z}^{\perp}$ and $g=\{\pi: i \leq N\} \in \mathcal{Z}$. Then,

$$
\langle g, \tilde{g}\rangle=\sum_{i \leq N} \int_{S} \pi \tilde{\pi} d Q .
$$

Let $B$ be a measurable set and let $g$ be such that for $k \neq i, j, \pi_{k}(s)=0$ for all $s \in S$, let for $s \in B, \pi_{i}(s)=-\pi_{j}(s)$ and let for $s^{\prime} \notin B, \pi_{i}\left(s^{\prime}\right)=\pi_{j}\left(s^{\prime}\right)=0$. Then,

$$
\begin{array}{r}
0=\langle g, \tilde{g}\rangle=\sum_{i \leq N} \int_{S} \pi_{i} \tilde{\pi}_{i} d Q, \\
\leftrightarrow \int_{B} \tilde{\pi}_{i}(s) d Q=\int_{B} \tilde{\pi}_{j}(a) d Q .
\end{array}
$$


Given that $B$ was arbitrary, it follows that $\pi_{i}=\pi_{j}$.

Parts 2 and 3: Observe that $g=M g+(g-M g)$. By the theory on linear operators, $K g=g-M g \in \mathcal{M}^{\perp}=\mathcal{K}$. To show uniqueness, let $\hat{g}, \hat{g}^{\prime} \in \mathcal{K}$ and $\tilde{g}, \tilde{g}^{\prime} \in \mathcal{M}$ and assume that $g=\hat{g}+\tilde{g}=\hat{g}^{\prime}+\tilde{g}^{\prime}$. Then,

$$
g-g=\hat{g}-\tilde{g}+\hat{g}^{\prime}-\tilde{g}^{\prime} .
$$

As $\mathcal{K}$ and $\mathcal{M}$ are vector spaces, $\hat{g}-\hat{g}^{\prime} \in \mathcal{M}$ and $\tilde{g}-\tilde{g}^{\prime} \in \mathcal{K}$, so,

$$
\|g-g\|^{2}=0=\left\|\hat{g}-\hat{g}^{\prime}\right\|^{2}+\left\|\tilde{g}-\tilde{g}^{\prime}\right\|^{2},
$$

which shows that $\hat{g}=\hat{g}^{\prime}$ and $\tilde{g}=\tilde{g}^{\prime}$. The proof of Part 3, i.e., the decomposition of $g$ into $Z g$ and $C g$, is similar and thus omitted.

Part 4: Let $g=\left\{\pi_{i}: i \leq N\right\}, K g=\left\{\tilde{\pi}_{i}: i \leq N\right\}$ and $M g=\left\{\hat{\pi}_{i}: i \leq N\right\}$. Then, by Part 2 ,

$$
\pi_{i}=\tilde{\pi}_{i}+\hat{\pi}_{i}
$$

Integrating over $S$ gives

$$
\int_{S} \pi_{i} d Q=\int_{S} \tilde{\pi}_{i} d Q+\int_{S} \hat{\pi}_{i} d Q=\int_{S} \tilde{\pi}_{i} d Q=c_{i} \int_{S} d Q
$$

where $\tilde{\pi}_{i}=c_{i} \in \mathbb{R}$. So,

$$
c_{i}=\tilde{\pi}_{i}=\frac{\int_{S} \pi_{i} d Q}{\int_{S} d Q} .
$$

Next, let $Z g=\left\{\hat{\pi}_{i}: i \leq N\right\}$ and $C g=\left\{\tilde{\pi}_{i}: i \leq N\right\}$. Then again,

$$
\pi_{i}=\hat{\pi}_{i}+\tilde{\pi}_{i} .
$$

Summing over all $i \leq N$ gives

$$
\sum_{j \leq N} \pi_{j}=\sum_{j \leq N} \hat{\pi}_{j}+\sum_{j \leq N} \tilde{\pi}_{j}=\sum_{j \leq N} \tilde{\pi}_{j} .
$$

Given that $C g \in \mathcal{C}$, i.e. $\tilde{\pi}_{i}=\tilde{\pi}_{j}$, we have,

$$
\tilde{\pi}_{i}=\frac{\sum_{j \leq N} \pi_{j}}{N} .
$$

Part 5: Consider $g=\left\{\pi_{i}: i \leq N\right\}$ then $K g=\left\{\tilde{\pi}_{i}: i \leq N\right\}$ is such that

$$
\tilde{\pi}_{i}=\frac{\int_{S} \pi_{i} d Q}{\int_{S} d Q},
$$

and therefore $C K g=\left\{\hat{\pi}_{i}: i \leq N\right\}$ is such that

$$
\hat{\pi}_{i}=\frac{\sum_{j \leq N} \tilde{\pi}_{j}}{N}=\frac{\sum_{j \leq N} \int_{S} \pi_{j} d Q}{N \int_{S} d Q} .
$$


Similarly, $K C g=\left\{\pi_{i}^{*}: i \leq N\right\}$ is such that

$$
\pi_{i}^{*}=\frac{\int_{S} \sum_{j \leq N} \pi_{j} d Q}{N \int_{S} d Q}
$$

By exchanging summation and integration, we obtain $C K g=K C g$.

Next, $C g=C(K g+M g)=C K g+C M g=K C g+C M g$. This implies that

$$
C M g=C g-K C g=M C g,
$$

which shows that $C$ and $M$ are also commutative. Next, $M g=Z M g+C M g=Z M g+$ $M C g$, so

$$
Z M g=M g-M C g=M(g-C g)=M Z g,
$$

which shows that $Z$ and $M$ also commute. Finally, $Z g=M Z g+K Z g=Z M g+K Z g$ so,

$$
K Z g=Z g-Z M g=Z(g-M g)=Z K g,
$$

which shows that $Z$ and $K$ commute.

Proof of Proposition 1. Under Assumption 3, there exist a function $f: \mathbb{R}_{+}^{2} \rightarrow \mathbb{R}$ such that on $\mathcal{M}$,

$$
\mathrm{CCI}(g)=f\left(\|g-Z g\|^{2},\|g-C g\|^{2}\right)=f\left(\|C g\|^{2},\|Z g\|^{2}\right) .
$$

Consider a game $g \in \mathcal{M}$ with $g \neq g_{0}$. Then if we use Assumption 2 with $\alpha=\frac{1}{\|g\|}$, we obtain

$$
\mathrm{CCI}(g)=\mathrm{CCI}(\alpha g)=\mathrm{CCI}\left(\frac{g}{\|g\|}\right)=f\left(\frac{\|C g\|^{2}}{\|g\|^{2}}, \frac{\|Z g\|^{2}}{\|g\|^{2}}\right),
$$

which follows from the fact that both $Z$ and $C$ are linear operators. Now,

$$
\frac{\|Z g\|^{2}}{\|g\|^{2}}+\frac{\|C g\|}{\|g\|^{2}}=1
$$

By defining $r(t)=f(1-t, t)$, we finally obtain that, for $g \in \mathcal{M}$ with $g \neq g_{0}$,

$$
\operatorname{CCI}(g)=r\left(\frac{\|Z g\|^{2}}{\|g\|^{2}}\right)=r\left(\frac{\|g-C g\|^{2}}{\|g-C g\|^{2}+\|g-Z g\|^{2}}\right) .
$$

Assumption 3 then implies that $r$ is strictly increasing in its argument.

In general, if $g \in \mathcal{G} \backslash \mathcal{K}$ then $g-K g=M g \neq g_{0}$. So, by Assumption 1 and the reasoning above,

$$
\mathrm{CCI}(g)=\mathrm{CCI}(M g)=r\left(\frac{\|M g-C M g\|^{2}}{\|M g-C M g\|^{2}+\|M g-Z M g\|^{2}}\right) .
$$

This establishes the result. 


\section{Appendix B: Additional Derivations for the Examples}

\section{Contests}

Tullock When $\alpha=3$, we obtain:

$$
\pi_{i}=\int_{0}^{v} \int_{0}^{v} \frac{1}{v} \frac{1}{v}\left(\left(\frac{x_{i}^{3}}{x_{i}^{3}+x_{j}^{3}}\right) v-x_{i}\right) \mathrm{d} x_{j} \mathrm{~d} x_{i}=0 .
$$

Thus, the game is already mean-normalized, i.e., $\bar{\pi}_{i}=0$. The calculation of our index yields:

$$
\operatorname{CCI}(g)=\frac{\left\|\pi_{1}\left(x_{1}, x_{2}\right)-\pi_{2}\left(x_{1}, x_{2}\right)\right\|^{2}}{2\left(\left\|\pi_{1}\left(x_{1}, x_{2}\right)\right\|^{2}+\left\|\pi_{2}\left(x_{1}, x_{2}\right)\right\|^{2}\right)} \approx \frac{0.15354}{0.32027} \approx 0.48
$$

Durham, Hirshleifer, and Smith We first need to mean-normalize the payoffs of the game by Durham, Hirshleifer, and Smith (1998). The average payoff is $\frac{v}{8}$. Thus, the mean-normalized payoff functions are:

$$
\pi_{1}-\bar{\pi}_{1}=\frac{x_{1}^{r}}{x_{1}^{r}+x_{2}^{r}}\left(v-x_{1}\right)\left(v-x_{2}\right)-\frac{v}{8} \text { and } \pi_{2}-\bar{\pi}_{2}=\frac{x_{2}^{r}}{x_{1}^{r}+x_{2}^{r}}\left(v-x_{1}\right)\left(v-x_{2}\right)-\frac{v}{8}
$$

We will henceforth set $v=20$ as in the experiment. For $r=1$, we obtain:

$$
\begin{aligned}
& \left\|\pi_{1}-\bar{\pi}_{1}-\left(\pi_{2}-\bar{\pi}_{2}\right)\right\|^{2}=\int_{0}^{20} \int_{0}^{20}\left(\frac{\left(x_{1}-x_{2}\right)}{x_{1}+x_{2}}\left(20-x_{1}\right)\left(20-x_{2}\right)\right)^{2} \mathrm{~d} x_{2} \mathrm{~d} x_{1} \approx 2.07342 * 10^{6} \\
& \left\|\pi_{1}-\bar{\pi}_{1}\right\|^{2}=\left\|\pi_{2}-\bar{\pi}_{2}\right\|^{2}=\int_{0}^{20} \int_{0}^{20}\left(\frac{x_{1}}{x_{1}+x_{2}}\left(20-x_{1}\right)\left(20-x_{2}\right)-\frac{20}{8}\right)^{2} \mathrm{~d} x_{2} \mathrm{~d} x_{1} \approx 2.198863 * 10^{6}
\end{aligned}
$$

Thus, we get $\mathrm{CCI}_{1,20}(g) \approx 0.236$ for the game with $r=1$ and $v=20$.

For $r=4$, we obtain:

$$
\begin{aligned}
& \left\|\pi_{1}-\bar{\pi}_{1}-\left(\pi_{2}-\bar{\pi}_{2}\right)\right\|^{2}=\int_{0}^{20} \int_{0}^{20}\left(\frac{\left(x_{1}^{4}-x_{2}^{4}\right)}{x_{1}^{4}+x_{2}^{4}}\left(20-x_{1}\right)\left(20-x_{2}\right)\right)^{2} \mathrm{~d} x_{2} \mathrm{~d} x_{1} \approx 5.13881 * 10^{6} \\
& \left\|\pi_{1}-\bar{\pi}_{1}\right\|^{2}=\left\|\pi_{2}-\bar{\pi}_{2}\right\|^{2}=\int_{0}^{20} \int_{0}^{20}\left(\frac{x_{1}^{4}}{x_{1}^{4}+y_{1}^{4}}\left(20-x_{1}\right)\left(20-x_{2}\right)-\frac{20}{8}\right)^{2} \mathrm{~d} x_{2} \mathrm{~d} x_{1} \approx 2.96298 * 10^{6}
\end{aligned}
$$

Thus, we get $\mathrm{CCI}_{4,20}(g) \approx 0.433$ for the game with $r=4$ and $v=20$.

Consistent with our prediction, contestants spend more resources on fighting in the experiment when $r=4$. This yields a more competitive outcome with lower payoffs. 


\section{Public Goods game}

We now provide the tedious calculation of our index that we left out in the main text. In general for an $N$-player game, the index is given by:

$$
\operatorname{CCI}(g)=\frac{\sum_{i=1}^{N}\left\|\pi_{i}-\bar{\pi}_{i}-\frac{1}{N}\left(\sum_{j=1}^{N}\left(\pi_{j}-\bar{\pi}_{j}\right)\right)\right\|^{2}}{\sum_{i=1}^{N}\left\|\pi_{i}-\bar{\pi}_{i}\right\|^{2}}
$$

where $\bar{\pi}_{i}$ is the mean payoff for player $i$. From the main text, recall that:

$$
\pi_{i}-\bar{\pi}_{i}=x_{i}-\alpha \sum_{j=1}^{N} x_{j}+\frac{\alpha N-1}{2} .
$$

Then:

$$
\begin{aligned}
& \pi_{i}-\bar{\pi}_{i}-\frac{1}{N} \sum_{j=1}^{N}\left(\pi_{j}-\bar{\pi}_{j}\right) \\
& =\frac{N-1}{N}\left[\left(\pi_{i}-\bar{\pi}_{i}\right)-\sum_{j \neq i}^{N} \frac{\pi_{j}-\bar{\pi}_{j}}{N-1}\right] \\
& =\frac{N-1}{N}\left[x_{i}-\alpha \sum_{j=1}^{N} x_{j}+\frac{\alpha N-1}{2}-\sum_{j \neq i}^{N} \frac{x_{j}-\alpha \sum_{k=1}^{N} x_{k}+\frac{\alpha N-1}{2}}{N-1}\right], \\
& =\frac{N-1}{N}\left[x_{i}-\sum_{j \neq i}^{N} \frac{x_{j}}{N-1}\right] .
\end{aligned}
$$

As such, for the numerator of our index, we obtain:

$$
\begin{aligned}
& \left\|\pi_{i}-\bar{\pi}_{i}-\frac{1}{N} \sum_{j=1}^{N}\left(\pi_{j}-\bar{\pi}_{j}\right)\right\|^{2} \\
& =\frac{(N-1)^{2}}{N^{2}} \int_{0}^{1} \ldots \int_{0}^{1}\left(x_{i}^{2}-2 x_{i} \sum_{j \neq i} \frac{x_{j}}{N-1}+\sum_{j \neq i} \frac{x_{j}^{2}}{(N-1)^{2}}+\sum_{j \neq i, k \neq i, j \neq k} \frac{x_{j} x_{k}}{(N-1)^{2}}\right) d x_{N} \ldots, d x_{1}, \\
& =\frac{(N-1)^{2}}{N^{2}}\left[\frac{1}{3}-2 \frac{1}{2} \frac{(N-1)}{(N-1)} \frac{1}{2}+(N-1) \frac{1}{3(N-1)^{2}}+\frac{(N-1)(N-2)}{4(N-1)^{2}}\right] \\
& =\frac{(N-1)^{2}}{N^{2}}\left[\frac{1}{3}-\frac{1}{2}+\frac{1}{3(N-1)}+\frac{(N-2)}{4(N-1)}\right] \\
& =\frac{(N-1)^{2}}{N^{2}}\left[\frac{1}{12(N-1)}(-2(N-1)+4+3(N-2))\right] \\
& =\frac{N-1}{12 N} .
\end{aligned}
$$


This shows that the numerator is independent of $\alpha$.

For the norms in the denominator, we obtain:

$$
\begin{aligned}
& \left\|\pi_{i}-\bar{\pi}_{i}\right\|^{2} \\
& =\int_{0}^{1} \ldots \int_{0}^{1}\left(x_{i}-\alpha \sum_{j=1}^{N} x_{j}+\frac{\alpha N-1}{2}\right)^{2} \mathrm{~d} x_{N} \ldots \mathrm{d} x_{1}, \\
& =\int_{0}^{1} \ldots \int_{0}^{1}\left(x_{i}^{2}-2 \alpha x_{i}^{2}-2 \alpha x_{i} \sum_{j \neq i} x_{j}+2 x_{i} \frac{\alpha N-1}{2}\right) \mathrm{d} x_{N} \ldots \mathrm{d} x_{1} \\
& +\int_{0}^{1} \ldots \int_{0}^{1}\left(\alpha^{2} \sum_{j=1}^{N} x_{j}^{2}+\alpha^{2} \sum_{j \neq k} x_{j} x_{k}-2 \alpha \sum_{j=1}^{N} x_{j} \frac{\alpha N-1}{2}\right) \mathrm{d} x_{N} \ldots \mathrm{d} x_{1} \\
& +\int_{0}^{1} \ldots \int_{0}^{1}\left(\frac{\alpha N-1}{2}\right)^{2} \mathrm{~d} x_{1} \ldots \mathrm{d} x_{N}, \\
& =\frac{1}{3}-\frac{2 \alpha}{3}-\frac{\alpha(N-1)}{2}+\frac{\alpha N-1}{2} \\
& +\frac{N \alpha^{2}}{3}+\frac{\alpha^{2}(N)(N-1)}{4}-\alpha N \frac{\alpha N-1}{2}+\left(\frac{\alpha N-1}{2}\right)^{2}, \\
& =\frac{1}{3}-\frac{2 \alpha}{3}-\frac{\alpha N}{2}+\frac{\alpha}{2}+\frac{\alpha N}{2}-\frac{1}{2} \\
& +\frac{N \alpha^{2}}{3}+\frac{\alpha^{2} N^{2}}{4}-\frac{\alpha^{2} N}{4}-\frac{\alpha^{2} N^{2}}{2}+\frac{\alpha N}{2}+\frac{\alpha^{2} N^{2}}{4}-\frac{\alpha N}{2}+\frac{1}{4}, \\
& =\frac{1}{12}\left(1-2 \alpha+N \alpha^{2}\right) .
\end{aligned}
$$

This is increasing in $\alpha$ for $\alpha>\frac{1}{N}$. Plugging in the two previous results, our index is given by:

$$
\begin{aligned}
\operatorname{CCI}(g) & =\frac{(N-1)^{2}}{N^{2}} \frac{N \frac{N}{12(N-1)}}{N \frac{1}{12}\left(1-2 \alpha+N \alpha^{2}\right)}, \\
& =\frac{(N-1)}{N} \frac{1}{\left(1-2 \alpha+N \alpha^{2}\right)}, \\
& =\frac{(N-1)}{(\alpha N-1)^{2}+(N-1)},
\end{aligned}
$$

which is the result that we use in the main text. 